\title{
Artículo de Revisión \\ Sífilis en el Siglo XXI: novedades en el enfrentamiento diagnóstico y terapéutico
}

\author{
Isabel Ogueta ${ }^{1}$, Elizabeth Valle ${ }^{1}$, Javier Contador ${ }^{2}$, Felix Fich $^{1}$
}

\section{RESUMEN}

La siffilis es una enfermedad de transmisión sexual altamente contagiosa con importantes complicaciones, pero con tratamiento efectivo en etapas tempranas. Actualmente, representa un problema de salud pública. La prevalencia reportada en EEUU desde el año 2008 es de 4,5 casos/100.000 habitantes, con una incidencia 10.6 millones de casos cada año, especialmente en hombres que mantienen relaciones sexuales con hombres (HSH) y pacientes portadores de VIH (PVIH). Los métodos diagnósticos basados en test moleculares aún no han sido validados para el diagnóstico de sífilis, lo que ha permitido establecer tres esquemas serológicos con diferentes rendimientos, según prevalencia poblacional. Desde este punto de vista, el screening reverso pareciera ser útil en población de alto riesgo, y el screening tradicional para la población general.
\end{abstract}

Palabras clave: sífilis, test no treponémico, screening reverso.

\section{SUMMARY}

Syphilis is a sexual transmitted disease highly contagious with important complications that can be prevented with an adequate treatment in early stages. Syphilis has become a public health issue, in the USA its incidence has increased from the 2001, with a rate in the 2008 of 4,5 cases/ 100000 people, with a greater prevalence in men who have sex with men (HSH) and people infected by HIV (PVIH). Despite molecular detection test are used for the diagnostic of many diseases, in syphilis we still using serologist test. There are three different schemes with different performance depending in the prevalence of syphilis in the population. In this setting reverse screening is the most adequate method for high prevalence versus traditional method that is better in general population.

Key words: syphilis; non treponemal test; reverse algorithm
${ }^{1}$ Departamento de Dermatología, Facultad de

Medicina, Pontificia

Universidad

Católica de Chile

${ }^{2}$ Escuela de

Medicina, Facultad

de Medicina,

Pontificia

Universidad

Católica de Chile.

Trabajo no recibió financiamiento.

Los autores declaran no tener conflictos

de interés.

Recibido el 13

de junio de 2019 ,

aceptado el 12 de

septiembre de 2019.

Correspondencia a:

Isabel Ogueta C.

Email:

iaogueta@uc.cl

Pontificia

Universidad

Católica de Chile.

Av Libertador

Bernardo

O'Higgins 340,

Santiago, Región

Metropolitana
$\mathrm{L}$ a Sífilis es una enfermedad de transmisión sexual con prevalencia global de un 0.5\% para hombres y mujeres entre 15-49 años en el año 2012, según la OMS ${ }^{1}$. Es causada por Treponema pallidum, bacteria perteneciente a la familia treponemataceae con transmisión principalmente por vía sexual y por vía trans-placentaria. Es una espiroqueta gram negativa, con un periodo de incubación que va desde las dos a las seis semanas, no cultivable, pero sí visible a la microscopía de campo oscuro y al microscopio de luz mediante gram y tinción de Giemsa². Clínicamente, se caracteriza por estadios clínicos de actividad intercalados con periodos de latencia. Se distingue la sífilis precoz que incluye la sífilis primaria, secundaria y latente precoz (duración de la infección menor de un año), y la sífilis tardía que incluye la latente tardía (duración de la infección mayor a un año) y terciaria ${ }^{3}$.

En Chile existe una prevalencia de 22.8/100.000 habitantes, con predominio en el sexo masculino (1,7:1), representando el 63\% del total de casos en el año 2016, siendo el principal grupo afectado el de 20-34 años. Con respecto al curso clínico de la enfermedad, la mayoría de los pacientes son detectados en sífilis precoz (51\%). En población de riesgo son frecuentes las co-infecciones, donde destaca la infección por VIH, presente en un $10 \%$, aumentando a $17 \%$ el año $2016 .{ }^{1}$ 
La evidencia internacional se asemeja a la experiencia nacional. En EEUU, el mayor reporte de infección por sífilis se alcanzó el año 1990, con su mayor baja el año 2000, re-emergiendo como un problema de salud pública desde el año 2001 en adelante. En el año 2008 , la prevalencia aumentó un 18,7\% con respecto a la cifra del año 2007, con una tasa de 4.5/100.000 habitantes, representando un $63 \%$ de los casos el grupo de pacientes HSH, a diferencia del año 2000, en que solo era un $4 \%$. Por el contrario, este aumento ha sido menor en las mujeres, con $36 \%$ de aumento en el 2008 con respecto al año $2007^{4}$, y se mantiene estable en el grupo de pacientes que mantienen relaciones sexuales exclusivamente con mujeres. Finalmente, la raza negra e hispana está 8 y 2 veces más afectada que la raza blanca, respectivamente. ${ }^{5}$

De este modo, un importante grupo de riesgo lo componen los pacientes portadores de VIH (PVIH), quienes actualmente representan un nuevo desafio, tanto del punto de vista diagnóstico como terapéutico, donde el 94\% de incidencia de infecciones de transmisión sexual (ITS) se da en este grupo y en HSH. Además, en este grupo, un $20 \%$ posee co-infección con otra ITS, principalmente Chlamydia rectal y Gonococo oro-faríngeo. Los dos principales factores de riesgo asociados son el abuso en el consumo de sustancias ilícitas y contar con más de cuatro parejas sexuales en los últimos seis meses. ${ }^{5}$

A la fecha, el diagnóstico se ha realizado mediante el uso de pruebas no treponémicas y treponémicas. Las primeras corresponden a reacciones antígenoanticuerpo que son usadas como métodos de tamizaje y seguimiento. Tanto el VDRL (Venereal Disease Research Laboratory) como el RPR (Rapid Plasma Reagin) miden anticuerpos (IgM / IgG) que reaccionan contra el material lipoidal liberado de las células dañadas del hospedero. Además, ambos detectan antígenos similares a los liberados por la bacteria, pero el primero posee afinidad por antígenos parecido a lipoproteínas y cardiolipinas; el segundo, a material proteico. ${ }^{6}$

Los test treponémicos detectan los anticuerpos específicos contra el Treponema pallidum, por lo que son más específicos y suelen ser usados como método confirmatorio. Entre ellos se encuentran: FTA-ABS (prueba de absorción de anticuerpos treponémicos fluorescentes), técnica de detección indirecta de an- ticuerpos; MHA-TP (ensayo de microhemaglutinación), técnica de hemoaglutinación pasiva con menor sensibilidad que el anterior durante la etapa precoz y tardía; Test de ELISA y de quimioluminiscencia, ambos inmunoensayos que detectan anticuerpos altamente específicos; Inmunocromatografía, técnica cualitativa basada en un sistema de reacciones inmunológicas; y, finalmente, el NAAT (técnica de amplificación de ácidos nucleicos) que se puede realizar con muestras de úlceras, sangre, mucosa y orina. Ésta última no estaría aprobada por la FDA. ${ }^{6,7}$

\section{Generalidades}

El cuadro clínico de la sífilis es variado, dependiendo de la etapa en que se encuentre al momento del diagnóstico. La sífilis primaria ocurre entre 1 a 90 días (promedio 21 días) desde la exposición. Se caracteriza por poseer un chancro de inoculación, el que se encuentra presente en el $40 \%$ de las infecciones, asociado o no a adenopatías inguinales. El chancro es una lesión elemental evolutiva que progresa de mácula a pápula y luego a úlcera. Es una lesión indolora de base indurada, fondo liso no purulento, bordes sellados, auto-resolutiva entre 1-6 semanas. La sífilis primaria presenta pruebas treponémicas positivas a partir de las 2 semanas y no treponémicas a las 3-4 semanas, por lo que el screening tradicional podría resultar falsamente negativo. ${ }^{8}$

La sífilis secundaria ocurre entre 3 a 6 meses después de la exposición y se le denomina la "gran simuladora", dado la diversidad de manifestaciones. La presentación clínica más característica es la roséola sifilítica. Este estadio se caracteriza por un exantema cutáneo no pruriginoso en tronco y extremidades con compromiso palmo-plantar. Puede haber presencia de adenopatías, afectación del estado general y fiebre, sin embargo, un tercio de los pacientes persiste con la lesión elemental al momento del diagnóstico. En este periodo existe un ascenso de los títulos ${ }^{8,9}$, con bacteremia e invasión de los tejidos. También, existen otras formas clínicas de secundarismo, como los condilomas planos, parches mucosos y parches alopécicos.

Por otro lado, la sífilis secundaria debe considerarse dentro del diagnóstico diferencial de verrugas anorectales. ${ }^{8}$ Los condilomas lata se diferencian de estas últimas por lo húmedo y aplanado de las lesiones, ya 
que contienen una alta carga de treponemas. En estos casos, cobra importancia el uso de técnicas de campo oscuro y las serologías.

El fenómeno prozona suele suceder en esta fase por exceso de treponemas y anticuerpos, favoreciendo una serología no treponémica falsamente negativa. Este fenómeno también puede presentarse en casos de neurosífilis, embarazo y coinfección con VIH. ${ }^{9}$

En la sífilis latente no existe manifestación de la enfermedad, pero posee serología positiva. Según el periodo de infección (menor o mayor a un año), se puede clasificar en latente precoz o tardía, respectivamente. Los pacientes pueden revertir su serología a "no reactivo", mantener títulos positivos o evolucionar a sífilis terciaria, la que se puede presentar hasta décadas luego de la infección8. En la fase terciaria se presentan lesiones muco-cutáneas, musculo-esqueléticas o parenquimatosas progresivas y destructivas, que pueden llevar a daño tisular irreversible ${ }^{2}$, además de aortitis o manifestaciones tardías del sistema nervioso central. ${ }^{8}$

Dada la alta prevalencia de sífilis en paciente PVIH, es importante tener un alto umbral de sospecha en estos pacientes. En primer lugar, cualquier lesión genital en PVIH debe hacer sospechar sífilis. ${ }^{5}$ En un $40 \%$ de los casos presentan chancros múltiples como infección primaria8. Además, la neurosífilis en PVIH se puede presentar en cualquiera de sus formas y estadio de la enfermedad, incluso con tratamiento precoz efectivo, siendo principalmente asintomática y con mayor riesgo en pacientes que poseen un conteo de CD4 (+) menor a 350 / uL, títulos de RPR mayores o iguales a 1:32 o VDRL mayor o igual a 1:16. El tratamiento para neurosífilis se indica con VDRL positivo en líquido cefalorraquídeo (LCR), pero dada su baja sensibilidad se podría indicar en casos que la celularidad del LGR fuese mayor a 20 cél $/ \mathrm{ml}^{.5}$

\section{ENFRENTAMIENTO DIAGNÓSTICO}

El Treponema pallidum no se puede cultivar y el uso de detección molecular como método diagnóstico es más bien acotado (por ejemplo, raspado de las lesiones, muestra de placenta, líquido cefalorraquídeo y suero), por lo que el método serológico es la principal herramienta diagnóstica. Sin embargo, este ha sido abordado desde distintos algoritmos, lo que implica diversas repercusiones clínicas y costos a nivel de salud pública. De esta manera, se han planteados tres posibles aproximaciones diagnósticas mediante el uso de serología: el método tradicional con test no treponémico y luego confirmación con test treponémico (Figura 1), el algoritmo reverso (Figura 2) y el algoritmo del Centro Europeo para la Prevención y Control de Enfermedades (EGDG) (Figura 3).

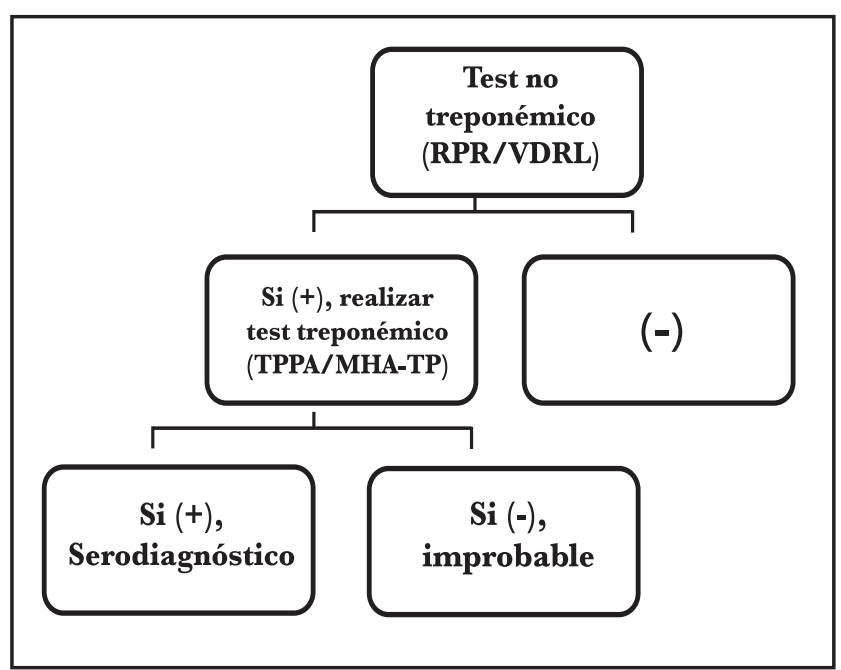

Figura 1

Método tradicional para el diagnóstico de Sífilis.

Abreviaturas: (+) resultado reactivo; (-) resultado no reactivo

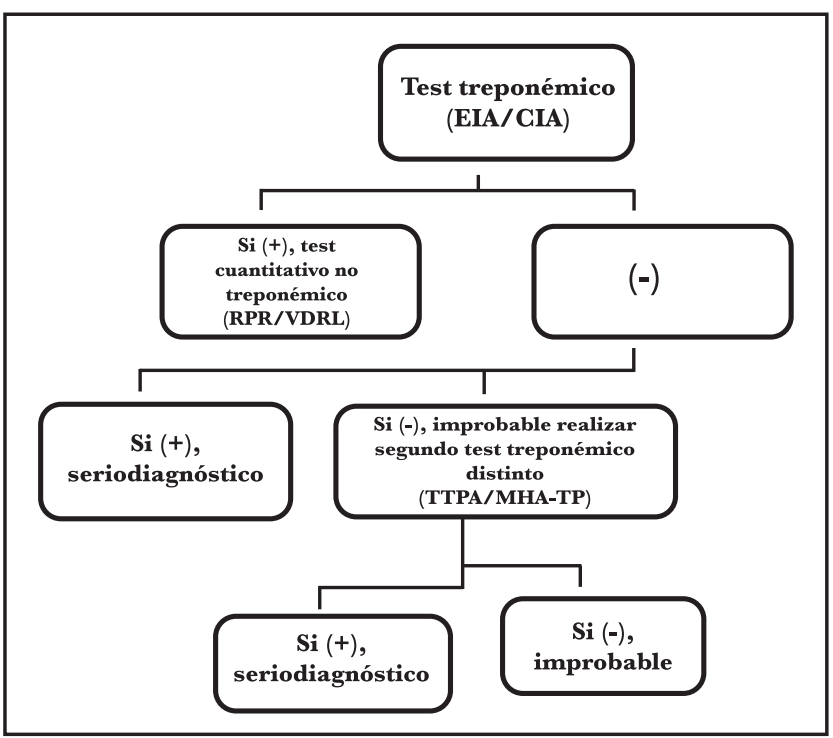

Figura 2

Algoritmo reverso para el diagnóstico de Sífilis.

Abreviaturas: (+) resultado reactivo; (-) resultado no reactivo 


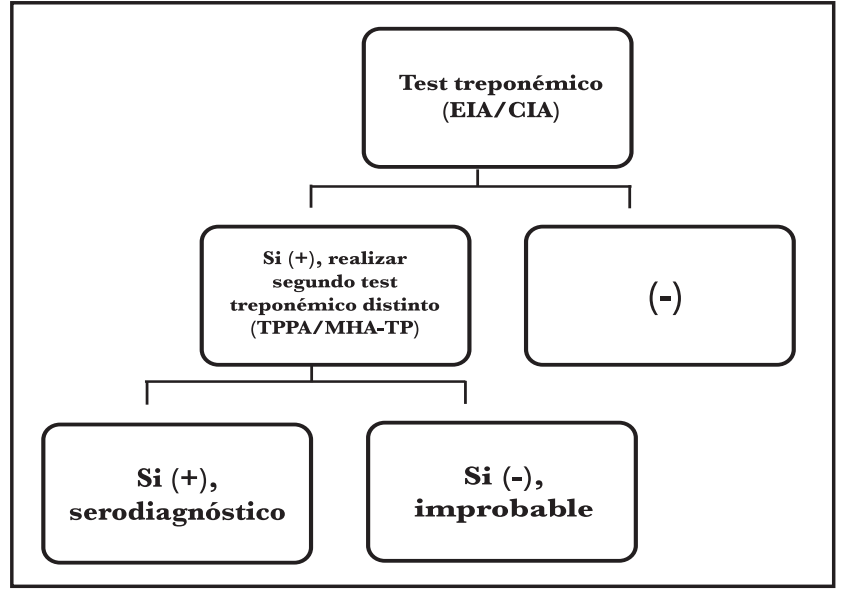

Figura 3

Algoritmo del Centro Europeo de Prevención y control de Enfermedades (ECDC), para el diagnóstico de Sífilis.

Abreviaturas: (+) resultado reactivo; (-) resultado no reactivo

\section{- Método tradicional}

Es el método actualmente recomendado por el CDC, en el que se comienza con un test no treponémico que identifica como antígeno la suspensión de cardiolipinas-colesterol y lectina de origen animal como resultado del daño celular generado por el Treponema pallidum en el hospedero, por lo que es un método indirecto de detectar infección, a diferencia de los test treponémicos, los cuales detectan antígenos directos del Treponema pallidum que no se sero-revierten luego del tratamiento antibiótico ni con el transcurso de la enfermedad, permaneciendo positivos de por vida. ${ }^{10}$

Los test no treponémicos pueden ser no reactivos en estadíos tempranos, tal como lo muestra un estudio en población de alta prevalencia, en donde el 24.2 $\%$ resultó con RPR (-)/TPPA (+), mediante técnica de aglutinación y CIA (inmunoanálisis por quimioluminiscencia), respectivamente. Así, el método tradicional poseería una sensibilidad de un $75.8 \%$ con el likelihood ratio (LR) más alto de los tres métodos $[0.24(>0.1)]$, lo que indica una alta probabilidad de resultados falsos negativos. ${ }^{11}$ Además, diversos estudios han demostrado que este método posee una falla diagnóstica en población de alta prevalencia de hasta un $30 \%$ cuando no se ha considerado el diagnóstico clínico previo. ${ }^{12}$ Sin embargo, en población de baja prevalencia resultaría ser costo-efectivo como medida de salud pública. ${ }^{13,14}$

\section{- Algoritmo reverso o screening reverso}

La secuencia reversa consiste en la realización de un test treponémico mediante el uso de las nuevas técnicas de enzimoinmunoanálisis (EIA/CIA), que buscan mayor rapidez y automatización, ya que los test no treponémicos siguen siendo pruebas manuales, operador dependiente. ${ }^{8}$ Según diversos estudios, comenzar con un test treponémico permite aumentar la sensibilidad en comparación al método tradicional. El estudio realizado por la CDG entre los años 2006 y 2010, mostró que un 56,7\% resultó reactivo mediante EIA/CIA, con RPR no reactivo. Entre los discordantes, el 31,6\% presentó TPPA (aglutinación de partículas; MHA-TP) o FTA-ABS negativos. De lo anterior se desprende que con el método reverso habría una reactividad 2.9 veces mayor en poblaciones con baja prevalencia de sífilis, lo que sugiere que habrían más falsos positivos. ${ }^{10}$ En un nuevo estudio de la CDC del año 2008, se revela una discordancia en el 50\% de las muestras, con un $17,2 \%$ de falsos positivos, lo que es contradictorio en población de alta prevalencia en donde CIA y TPPA poseen un valor kappa de 0.953 (concordancia de un 97.7\%, IC 95\%), siendo CIA $100 \%$ sensible con una especificidad de un $98.8 \%$ al compararlo con la reactividad del TPPA como gold estándar. ${ }^{12,15,16}$

En cuanto a lo anterior, es importante considerar que todo test logra mantener su sensibilidad, pero el valor predictivo positivo será distinto (VPP) dependiendo de la prevalencia poblacional de la enfermedad, siendo este valor mayor a mayor prevalencia de la patología. Así, cuando el screening reverso es aplicado en poblaciones de riesgo, pero de baja prevalencia; como dadores de sangre y embarazadas, puede resultar en un menor VPP. ${ }^{15,17}$

A pesar de los falsos positivos reportados en población de baja prevalencia, el algoritmo reverso es un método cada vez más usado. Presenta como gran inconveniente la serodiscordancia entre los test treponémicos y los no treponémicos, la que puede deberse a: sífilis primaria en persona que aún no desarrolla anticuerpos, sífilis previa tratada, falso positivo o efecto de prozona. Dado lo anterior, se debe realizar un segundo test treponémico con técnica distinta a EIA/CIA. Se sugiere usar el TPPA (MHA-TP) como test confirmatorio, a pesar que el FTA-ABS es considerado el 
gold estándar en muchas instituciones. Esto, debido a que el segundo test posee menor especificidad, menor sensibilidad y requiere personal y equipos entrenados. ${ }^{10,18}$

Un segundo test treponémico negativo indica una baja probabilidad de infección por sífilis. Sin embargo, un segundo test positivo posee dos posibles interpretaciones: por una parte, si existe el antecedente de sífilis tratada no hay necesidad de indicar un nuevo tratamiento a excepción de que se sospeche reinfección. Ante la ausencia de antecedente se deben abordar los casos de manera clínica y tratar como sífilis de duración indeterminada ante signos y síntomas de infección. Importante agregar que este enfrentamiento diagnóstico no resultaría útil en caso de madre discordante respecto de transmisión vertical ni en predecir la evolución a sífilis terciaria, neurosífilis y al compromiso oftálmico. ${ }^{14}$

\section{- Algoritmo del Centro Europeo de preven- ción y control de enfermedades (EGDC)}

El esquema comienza con pruebas treponémicas (EIA/CIA), seguido por una prueba treponémica distinta (por lo general, TPPA), sin necesidad de pruebas no trepónemicas. Según la literatura disponible, esta sería la mejor opción frente a población de alta prevalencia de sífilis, alcanzando una sensibilidad de $99.85 \%$, con una especificidad del 100\%, superando incluso al método de screening reverso, ya que sería innecesario un test no treponémico para el diagnóstico. ${ }^{12}$ A pesar de lo anterior, las pruebas no treponémicas siguen siendo utilizadas por su valor en el seguimiento de los pacientes y evaluar la respuesta a tratamiento.

\section{Propuesta}

Dado los resultados, es fácil considerar que el screeening reverso o el ECDC son los métodos de elección cuando se posee una alta prevalencia de la enfermedad, y que el método reverso presenta altos resultados falsos positivos cuando la prevalencia de la enfermedad es baja. En vista de lo anterior, no sugerimos realizar screening reverso a modo de tamizaje universal, pero sí frente a una alta prevalencia de la enfermedad.
Cabe recordar que en estudios de población de alta prevalencia se encontró 6.3 veces más reactividad mediante EIA/CIA (14.5\%) en comparación a la población de baja prevalencia $(2.3 \%)$. El porcentaje de serodiscordancia fue mayor en la población de baja prevalencia en comparación a la de alta prevalencia (60.6\% vs. $50.6 \%$, respectivamente). Dentro del grupo con discordancia, el porcentaje de falsos positivos fue de un $14.1 \%$ (alta prevalencia) en comparación al 40.8\% en población de baja prevalencia; es decir, 2.9 veces mayor en el segundo grupo ${ }^{10}$ (Tabla 1). Lo anterior es concordante con estudios realizados en población de alta prevalencia que muestran que el método tradicional posee una sensibilidad global de un $75.81 \%$, con una sensibilidad para la detección de sífilis primaria y terciaria de un $75 \%$ y $67.81 \%$, respectivamente (Tabla 2). En comparación, el screening reverso y el algoritmo del ECDC poseen mayor sensibilidad (99.85\%) y especificidad global (99.98\%) para el serodiagnóstico de sífilis en población de alta prevalencia (independiente del estadío de la enfermedad), presentando una alta concordancia entre los diferentes estudios (99,98\%; valor kappa de 0.996). ${ }^{11}$ Sin embargo, es el test no treponémico el que permite realizar seguimiento y evaluar la respuesta a tratamiento.

Dado lo anterior, no es recomendable el uso del método reverso como screening universal, ya que los estudios en poblaciones de baja prevalencia (población general), han demostrado gran porcentaje de falsos positivos con dudas al momento del tratamiento frente a la presencia de serodiscordancia. Por ejemplo, un estudio de la CDC en población general dio cuenta de un $56.7 \%$ de falta de reactividad con test no treponémicos en individuos que poseían un screening inicial mediante EIA/CIA reactivo. ${ }^{10}$ Así, se podría recomendar realizar un screening dicotómico o mixto (Figura 4), en donde se ejecute el método reverso en población de riesgo o alta sospecha clínica (Tabla 3), ya que esto permitiría mejorar el valor predictivo positivo con un mismo test y usar el método tradicional en población general, tal como sugiere la CDC.

Se podría realizar un primer test treponémico como método de screening en población de riesgo), que en caso de ser reactivo requeriría de un segundo test no treponémico (RPR o VDRL). Finalmente, según el 
Tabla 1

Adaptado de "Central Disease Control and Prevention. Discordant Results from Reverse Sequence Syphilis Screening Five Laboratories, United States, 2006-2010”.MMWR 2011. 60 (5); 142-133.

\begin{tabular}{l|c|c|c|c}
\hline & Total de individuos & EIA /CIA reactivo & RPR no reactivo & $\begin{array}{c}\text { FTA-ABS / TP- } \\
\text { PA no reactivo }\end{array}$ \\
\hline $\begin{array}{l}\text { Baja } \\
\text { prevalencia }\end{array}$ & 127402 & $2.3 \%$ & $60.6 \%$ & $40.8 \%$ \\
Alta & & 2984 individuos & 1807 Individuos & 737 individuos \\
prevalencia & 12774 & $14.5 \%$ & $50.6 \%$ & $14.1 \%$ \\
$\begin{array}{l}\text { Población } \\
\text { general }\end{array}$ & 140176 & 1850 individuos & 936 individuos & 129 individuos \\
\end{tabular}

Tabla 2

Adaptado de Man-Li Tong,et al. Analysis of 3 Algorithms for Syphilis Serodiagnosis and Implications for Clinical Management Clinical Infectious Diseases 2014;58(8):1116-24

\begin{tabular}{lllll}
$\begin{array}{l}\text { Diagnóstico: } \\
\text { Serodiagnóstico }\end{array}$ & Muestras & Tradicional & Reverso & ECDC \\
\hline Sífilis Primaria & 24 & $75 \%$ & $95.8 \%$ & $100 \%$ \\
Sífilis Secundaria & 365 & $99.1 \%$ & $100 \%$ & $99.7 \%$ \\
Precoz & 198 & $78.2 \%$ & $100 \%$ & $98.48 \%$ \\
Tardía & 1578 & $73.07 \%$ & $98.87 \%$ & $99.4 \%$ \\
Terciaria & 584 & $67.8 \%$ & $99.83 \%$ & $99.3 \%$ \\
Total & 2794 & $75.8 \%$ & $99.85 \%$ & $99.38 \%$ \\
\hline
\end{tabular}

Tabla 3

Población de alto riesgo, susceptible a ser sometida al esquema dicotómico de detección de sífilis.

\section{POBLACIÓN DE ALTO RIESGO, 21, 22, 23,24}

- PHIV

- HSH : OR 3.43

Principalmente entre 20-34 años ( Chile) / 20-29 años en EEUU ( Prevalencia más alta en 2014)

- Mujer que mantiene relaciones sexuales (RS) con HSH

- Autoreporte ITS/ presencia de otras ITS : OR 7.24

- Conductas de riesgo: RS grupales, RS sin protección con más de una pareja, individuos que intercambien sexo por drogas o dinero, RS en estado de intoxicación, etc.

- Mayor o igual a 4 parejas sexuales en un periodo de 6 meses.

- Abuso de sustancias / Uso de drogas endovenosas : OR 2.37

- Sexo con usuario de drogas endovenosas

- Pareja con sífilis temprana

- Historia de encarcelamiento

- Trabajadora sexual

- Inicio precoz ( $<15$ años)

*Población con bajo riesgo de infección: embarazada, dadores de sangre. 


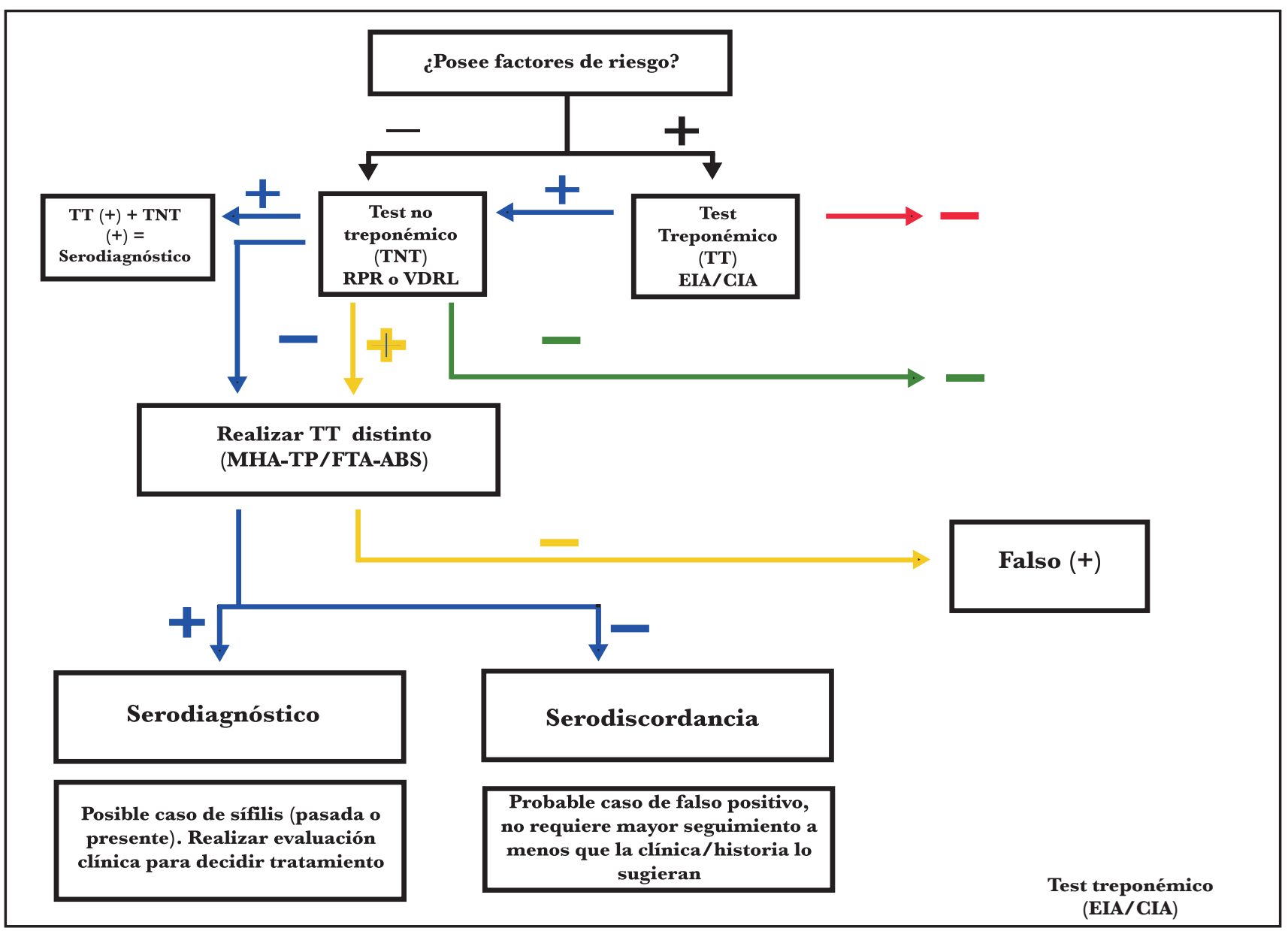

\section{Figura 4}

Método dicotómico o mixto propuesto para el diagnóstico de sífilis, según riesgo poblacional.

Abreviaturas: CIA: Inmunoensayo por quimioluminiscencia; EIA: Enzimoinmunoanálisis; TT: Test treponémico; TNT: Test no treponémico; TPPA: Ensayo de aglutinación de partículas de Treponema pallidum; FTA-ABS: prueba de absorción de anticuerpos treponémicos fluorescentes; MHA-TP: ensayo de microhemaglutinación; (+) resultado reactivo; (-) resultado no reactivo.

resultado de éste se podría confirmar la infección o se necesitaría de otro test treponémico distinto al primero para lograr determinar una conducta (Figura 4). En cambio, en población de bajo riesgo se podría iniciar el tamizaje mediante el uso de un test no treponémico. En ambos casos, el seguimiento se realizaría mediante este último test serológico.

\section{Novedades}

Dentro de los nuevos avances, destacan aparatos conectados a Smartphones que permiten obtener ELISA triple para VIH, anticuerpos treponémicos y anticuerpos no treponémicos, y el año 2002 se aprobó ELISA en suero o plasma como método de tamizaje, lo que permitiría facilitar el uso del método reverso. ${ }^{19,20}$ Sin embargo, poseen amplias desventajas: no permiten realizar titulación y la mayoría corresponden a test treponémicos, impidiendo así el seguimiento y la evaluación de respuesta a tratamiento. Podrían constituirse como una herramienta útil para el screening antenatal en lugares rurales alejados o como una nueva técnica a usar en muestras de LCR en sitios con recursos limitados. ${ }^{14,20}$

El VDRL en LGR es la prueba más específica para el diagnóstico de neurosífilis, pero con una sensibilidad del 50\%14. El RPR es menos sensible que el VDRL en LCR y no debe utilizarse para el diagnóstico de neurosífilis. La pleocitosis del LCR en la sífilis es predominante de linfocitos, y un valor mayor o igual a 5 células/ml se considera predictor de enfermedad; sin embargo, su especificidad puede ser menor en PVIH. 
Finalmente, han sido descritos nuevos exámenes diagnósticos de neurosífilis. Se ha evaluado el test para CXCL-13 en LGR, un receptor de células B que posee buena relación con el VDRL del LCR en pacientes con VIH. ${ }^{14}$

\section{Tratamiento}

El tratamiento de elección según la CDC y las guías europeas sigue siendo 1 dosis de 2,4 millones de unidades de Pencilina Benzatina intramuscular para la sífilis primaria, secundaria y formas latentes menores de un año. En cambio, para las formas tardías (latentes mayores de uno año, afección cardiovascular, goma sifílico, etc.), se recomienda Penicilina Benzatina 2,4 millones de unidades intramuscular semanales por 3 semanas consecutivas. En caso de contraindicación, se recomienda el uso de tetraciclinas y cefalosporinas, ya que se debe considerar que la resistencia a los macrólidos va en aumento. La guía americana recomienda tratar con dosis única en pacientes VIH con sífilis en las formas precoces, aunque la literatura sigue siendo contradictoria. ${ }^{14}$ En Chile, en sífilis primaria, secundaria y latente precoz, se indican 2,4 millones de unidades de Penicilina Benzatina intramuscular semanal por 2 semanas consecutivas. En sífilis latente tardía y terciaria el tratamiento se realiza por 3 semanas consecutivas. En los alérgicos a la penicilina, se recomienda doxiciclina o tetraciclina por 15 ó 30 días según el estadío clínico de la enfermedad. ${ }^{25}$

El éxito del tratamiento se define con caída de dos diluciones o la disminución en 4 veces de los títulos de los test no treponémicos. El seguimiento se realiza siempre al primer mes del término del tratamiento y, según el paciente es PVIH o no, se continúa cada 3 ó 6 meses, respectivamente. ${ }^{25}$ Lo anterior conlleva implicancias en el tratamiento, ya que una disminución inadecuada de los títulos nos debe hacer plantear la posibilidad de una punción lumbar. Lo anterior, ya que en la imposibilidad de plantear una reinfección, el paciente puede estar presentando una neurosífilis. Dentro de las causas de una respuesta serológica desfavorable, se encuentran: reinfección, tiempo de evolución (por ejemplo, sífilis tardía), VIH concomitante (especialmente sin tratamiento anti-retroviral), bajos títulos de RPR/VDRL al momento del diagnóstico en pacientes con sífilis precoz o presencia de neurosifilis. ${ }^{26,27}$

En cuanto a la duración, el tratamiento mínimo en el caso de sífilis tardía requiere de la mantención de una concentración de $0,018 \mathrm{mcg} / \mathrm{ml}(0.03 \mathrm{IU})$ por un periodo de tres semanas, lo que se logra con una dosis de Penicilina Benzatina 2.4 millones intramuscular semanal por tres semanas. ${ }^{26}$ En el caso que realizada la terapia no exista una baja adecuada de los títulos (concepto de "serofast" en la literatura anglosajona), se recomienda repetir una dosis de Penicilina Benzatina a los seis meses, sin existir una recomendación de hacer uso de mayores dosis en PVIH en relación a pacientes seronegativos. ${ }^{21}$ Lo anterior, se correlaciona con la recomendación actual de la CDC de seguimiento de pacientes sin infección por VIH, a los 6-12 meses y en PVIH a los 3-6-9-12 meses, ya que en vista de este riesgo de serofast es importante evaluar continuamente la necesidad de un segundo ciclo de de antibioterapia. ${ }^{26}$

La segunda opción de tratamiento es la doxiciclina, sin embargo, ceftriaxona (cefalosporina de tercera generación con gran penetración en el $\mathrm{SNC}$ ), ha demostrado ser igual de eficaz en relación a serología residual, tasa de recaídas en el tratamiento y, además, alcanzaría mayor concentración en LGR que la Penicilina Benzatina, por lo que sería una buena alternativa para el tratamiento de la neurosífilis. Se ha demostrado que el tratamiento con ceftriaxona a los 3, 6 y 12 meses posee igual respuesta serológica, definida como una disminución de cuatro veces de los títulos sin un aumento en el periodo de seguimiento. A pesar de lo anterior, es necesaria mayor evidencia para respaldar la no diferencia en relación a la tasa de éxito y fracaso entre ambos esquemas antibióticos, ya que ceftriaxona posee mayores tasas de resistencia. El uso de Penicilina Benzatina sigue siendo la primera línea de tratamiento, sin sustitutos actuales ${ }^{28,29,30}$, pero, hay que considerar que un $10 \%$ de los pacientes posee alergia a la penicilina y que un 3-7\% de los individuos posee alergia cruzada con cefalosporinas. ${ }^{26}$

Una nueva metodología utilizada es el tratamiento fortificado con Penicilina Benzatina a dosis clásica, seguido por ceftriaxona $1 \mathrm{gr} /$ día por 10 días y posteriormente con doxiciclina $100 \mathrm{mg}$ cada $12 \mathrm{hrs}$ por 20 días, lo que ha logrado mejores resultados que el 
grupo con tratamiento clásico, sin aumentar los efectos adversos, con una tasa de éxito de un $100 \%$ en el grupo con tratamiento fortificado. Además, este grupo no presentó complicaciones en comparación al grupo control con tratamiento estándar, que tuvo un éxito de un $68 \%$. Esta nueva propuesta podría ser planteada como un nuevo esquema de tratamiento, sin embargo no es el recomendado por la CDC, pero sería prudente considerarlo, ya que un 20\% (y hasta un $60 \%$ ) de pacientes PVIH posee neurosífilis en estadíos precoces, y la ceftriaxona y doxiciclina son dos antibióticos que alcanzan una CIM adecuada para Treponema pallidum a nivel del sistema nervioso central, en comparación a la penicilina. ${ }^{26}$

Finalmente, respecto al tratamiento, se ha evaluado el uso profiláctico de doxiciclina diaria, sin embargo, no ha demostrado cambios en el desarrollo de sífilis, pero sí en la adquisición de otras ITS. La única intervención que ha demostrado disminución del riesgo de infección por Treponema pallidum, es la circuncisión masculina. ${ }^{14}$

\section{Conclusiones}

La sífilis es una enfermedad vigente, con mayor incidencia en HSH y en PVIH. La utilidad del screening reverso sigue siendo discutible y se aplica según la población afectada. Así, parece razonable realizar screening reverso en población con alta prevalencia, ya que el algoritmo presentaría un $100 \%$ de sensibilidad con una especificidad de un 98.8\% versus el método tradicional que podría no diagnosticar más de un cuarto de los casos. Sin embargo, no es recomendable en población general, dado que no sería costo-efectivo en términos de seguimiento y presentaría una alta incidencia de falsos positivos. Se podría iniciar con test ELISA treponémico y confirmar con FTA-ABS/ MHA-TP, ya que los falsos positivos se dan principalmente en pacientes PVIH, que tienen un alto compromiso inmunológico, con deterioro de la inmunidad celular, prevaleciendo la humoral. El tratamiento de elección sigue siendo el uso de Penicilina Benzatina, con las dosis y las frecuencias recomendadas por la CDC o según las normas propias de cada país, sin embargo, en algunos casos seleccionados, se pueden considerar otras alternativas terapéuticas.

\section{REFERENCIAS}

1. 1. Cáceres K. Situación epidemiológica de sífilis (CIE 10: A50-A53.9). Chile, 2016. Revista Chilena de Infectología 2018; 35 (3): 284-296

2. Sekikawa, Y., Oda, R., Hongo, I., \& Ono, E. The Diagnosis of Secondary Syphilis by Gram and Giemsa Staining. Internal medicine (Tokyo, Japan), 2017; 56 (15) 20-83.

3. Lukehart S, Kasper D, Fauci A, Hauser S, Dan Longo J, Jameson L, Loscalzo J. Capítulo 206: Sífilis. Harrison principios de medicina interna. Vol 2. 19a edition. McGraw-Hill; 2016.

4. Norris, S. Changing Algorithms in Syphilis Laboratory Diagnosis. Clinical Microbiology Newsletter 2010; 32(8):59-64

5. Marrazzo, J. What's New in Sexually Transmitted Infections in the HIV Care Setting: Focus on Syphilis and Gonorrhea. Top Antivir Med. 2015;22(5):698-701

6. Diaz, J. Vigilancia epidemiológica de sífilis y gonorrea. Rev Chilena Infectol 2013; 30 (3): 303-310

7. Golden, M. M O'Donnell. ,S Lukehar. Treponema pallidum Nucleic Acid Amplification Testing to Augment Syphilis Screening Among Men Who Have Sex with Men. J Clin Microbiol. 2019 Jun 12. pii: JCM.00572-19

8. Cherneskie, T. An Update and Review of the Diagnosis and Management of Syphilis. Region II STD/VIH Prevention Training Center; New York City Department of Health and Mental Hygiene, New York, NY: 2006.

9. Liu LL, Lin LR, Tong ML, et al. Incidence and risk factors for the prozone phenomenon in serologic testing for syphilis in a large cohort. Clin Infect Dis 2014; 59:384

10. Central Disease Control and Prevention. Discordant Results from Reverse Sequence Syphilis Screening Five Laboratories, United States, 2006-2010. MMWR 201160 (5) 142-133

11. Man-Li Tong,Li-Rong Lin,Li-Li Liu, Analysis of 3 Algorithms for Syphilis Serodiagnosis and Implications for Clinical Management Clinical Infectious Diseases 2014;58(8):1116-24

12. Simčič et al. Serological diagnosis of syphilis: a comparison of different diagnostic methods. Acta Dermatovenerol APA 2015;24:1720

13. Muhammad G. Morshed, Ameeta E. Singh. Recent Trends in the Serologic Diagnosis of Syphilis. Clinical and Vaccine Immunology 2015; 22:137-147.

14. Tuddenham, S Ghanem, K. Emerging trends and persistent challenges in the management of adult syphilis BMC Infectious Diseases (2015) 15:351

15. Hee Jin Huh, Jae-Woo Chung. Comparison of Automated Treponemal and Nontreponemal Test Algorithms as First-Line Syphilis Screening Assays Ann Lab Med 2016;36:23-27

16. Matthew J. Which algorithm should be used to screen for syphilis? Curr Opin Infect Dis 2012, 25:79-85

17. Morshed MG. Current trend on syphilis diagnosis: issues and challenges. Adv Exp Med Biol. 2014;808:51-64.

18. Tsang R, Radons S, Morshed M. Laboratory diagnosis of syphilis: A survey to examine the range of tests used in Canada. Can J Infect Dis Med Microbiol 2011;22(3):83-87. tamizaje 
19. Food and Drug Administration.Complete List of Donor Screening Assays for Infectious Agents and HIV Diagnostic Assays [Internet]. Disponible en: https://www.fda.gov/vaccines-blood-biologics/ blood-donor-screening/complete-list-donor-screening-assaysinfectious-agents-and-hiv-diagnostic-assays\#anti_Tpallidum_Assays

20. Hicks Ch. Syphilis: Screening and diagnostic testing. UpToDate Marrazo, J [Internet]. Disponible en: https://www.uptodate.com/ contents/syphilis-screening-and-diagnostic-testing?

21. Rapone da Motta,L . Dea Sperhacke, R, de Gregori Adami, Syphilis prevalence and risk factors among young men presenting to the Brazilian Army in 2016 A da Motta et al. Medicine (2018) 97:47

22. Trivedi et al. National Trends and Reported Risk Factors Among Pregnant Women With Syphilis in the United States, 2012-2016. Obstetric Gynecology. 2019 Jan;133(1):27-32

23. Ghassabi F, Malekzadegan Y, Sedigh Ebrahim-Saraie H, et al Frequency of gonorrhea and syphilis co-infection and related risk factors in HIV patients from Shiraz, South of Iran. Caspian J Intern Med 2018; 9(4): 397-402

24. Nishijima T, Teruya K, Shibata S, Yanagawa Y, Kobayashi T, Mizushima D, et al. (2016) Incidence and Risk Factors for Incident Syphilis among HIV-1-Infected Men Who Have Sex with Men in a Large Urban HIV Clinic in Tokyo,2008-2015. PLoS ONE 11(12): e0168642. doi:10.1371/journal.pone.0168642
25. Normas de Profilaxis, diagnóstico y tratamiento de las Infecciones de transmisión sexual (ITS) 2016. Departamento Programa Nacional de Prevención y Control del VIH/SIDA e ITS. Ministerio de Salud. Disponible en www.minsal.cl

26. Ghanem, K Management of Adult Syphilis: Key Questions to Inform the 2015 Centers for Disease Control and Prevention Sexually Transmitted Diseases Treatment Guidelines Clinical Infectious Diseases 2015;61(S8):S818-36

27. Drago F, Ciccarese G, Broccolo F, Sartoris G, Stura P, Esposito S, Parodi A. A new enhanced antibiotic treatment for early and late syphilis. Journal of Global Antimicrobial Resistance. 2016; 5:64-66

28. Zhen Lianga, Ya-Ping Chenb, Chun-Sheng Yangc, et al. ReviewMetaanalysis of ceftriaxone compared with penicillin for thetreatment of syphilis.International Journal of Antimicrobial Agents (2015) 47 6-11

29. Ros-Vivancos, C González-Hernández, M, Sánchez-Payá J et al. Evolución del tratamiento de la sífilis a lo largo de la historia.Rev Esp Quimioter 2018;31(6): 485-492

30. L. V. Stamm,. Syphilis: antibiotic treatment and resistance.Epidemiol. Infect. (2015) 143, 1567-157 